\title{
En busca de equilibro entre la regulación y la autorregulación de la publicidad comportamental en línea
}

\author{
Esther MARTÍNEZ PASTOR \\ Universidad Rey Juan Carlos de Madrid \\ esther.martinez.pastor@urjc.es \\ Mercedes MuÑOZ SALDAÑA \\ Universidad de Navarra \\ mms@unav.es
}

Recibido: 28/11/2012

Aceptado: 23/01/2013

\begin{abstract}
Resumen
La publicidad comportamental en línea es la apuesta del mercado publicitario al vertiginoso crecimiento de la industria en la red. La instalación y uso de las cookies en los dispositivos de los usuarios es lo que posibilita esta práctica comercial, pero, al mismo tiempo, pone en jaque derechos de los usuarios como el control de sus datos personales o su derecho a la intimidad. Las políticas europeas han respondido a esta controversia poniendo el acento en el principio del consentimiento previo (opt-in), sin embargo, las recientes iniciativas de autorregulación puestas en marcha por la industria e inspiradas por el planteamiento estadounidense, no parecen respetar del todo este principio. Resulta evidente la necesidad de un acuerdo y equilibrio ente los intereses públicos y privados implicados en este conflicto.

Palabras clave: Publicidad comportamental en línea, regulación, autorregulación, datos personales, intimidad.

\section{In Search of Balance between Regulation and Self-regulation of Online Behavioral Advertising}

\begin{abstract}
Behavioral advertising is the advertising market bet the rapid growth of the industry in the network. The installation and use of cookies on users' devices allows this commercial practice, and at the same time, they have the rights of users and control their personal data or privacy. European policies have responded to this problem with the principle of prior consent (opt-in) against recent industry initiatives and inspired by American ideas of self-regulation. There is clearly a need for an agency agreement and balance public and private interests involved in this conflict.

Keywords: Behavioral advertising, on line, regulation, self-regulation, personal data, privacy.

Referencia normalizada

MARTÍNEZ PASTOR Esther y MUÑOZ SALDAÑA, Mercedes (2013): "En busca de equilibro entre la regulación y la autorregulación de la publicidad comportamental en línea". Estudios sobre el Mensaje Periodístico. Vol. 19, Núm. especial marzo, págs.: 289-297. Madrid, Servicio de Publicaciones de la Universidad Complutense.
\end{abstract}

Sumario. 1. Introducción. Dos posturas frente a las cookies. 2. Metodología. 3. Regulación jurídica de la publicidad comportamental en línea. 4. Autorregulación de la publicidad comportamental en línea. 5. Conclusiones. Un apunte final 6. Referencias bibliográficas.

\section{Introducción. Dos posturas frente a las cookies}

El desarrollo de los medios digitales ha impulsado la aparición de nuevos formatos publicitarios como la publicidad comportamental online (online behavioural adverti- 
sing). A través de este modelo las marcas apuestan por una publicidad dirigida a un público específico llegando a ser personalizada. Este tipo de publicidad se caracteriza por instalar cookies para obtener información de visitas realizadas anteriormente a una misma página web. Las cookies son generadas por páginas web y almacenadas en el ordenador del usuario. Éstas pueden recopilar información durante la visita a la página, como nombres de usuario o direcciones de correo, la cual puede ser vendida a gestores de publicidad personalizada. Las cookies no permiten conocer la identidad concreta del usuario pero permiten registrar el comportamiento online que se ha llevado a cabo desde un determinado ordenador y navegador. Sin embargo, un alto porcentaje de usuarios desconocen que su comportamiento en la red está siendo seguido mediante cookies ni tienen conocimiento de quién lo hace o con qué finalidad. Por este motivo se cuestiona si este tipo de publicidad vulnera el derecho de los usuarios respecto de la utilización de sus datos personales o la salvaguarda de su intimidad en la navegación. Ante esta realidad se podría afirmar que hay dos posturas contrapuestas: una, que aboga por una protección jurídica de los derechos de los usuarios y, otra, que es partidaria de aprovechar las infinitas posibilidades que ofrece la red. Sin embargo, el futuro exige posturas equilibradas que faciliten el desarrollo de la publicidad online, especialmente por su trascendencia económica, y al mismo tiempo, que respeten los derechos de los usuarios en Internet.

La intensidad legislativa a este respecto en los últimos años es un reflejo de la importancia que las instancias políticas conceden a este difícil reto. Desde la Unión Europea cabe destacar la reciente Directiva 2009/136/CE sobre privacidad las comunicaciones electrónicas (art. 5.3.) y las aportaciones el Grupo de Trabajo del artículo 29 (GT 29) sobre la publicidad comportamental. En el ámbito nacional la última aportación legislativa es el Real Decreto-ley 13/2012, de 30 de marzo por el que se transponen directivas en materia de mercados interiores de electricidad y gas y en materia de comunicaciones electrónicas. Como complemento del marco legal, destacan iniciativas de la industria como la Self regulatory principles for online behavioral advertising (FTC, 2009) y el Código de Autorregulación Sobre las Buenas Prácticas de la Online Behavioural Advertising (EASA/IAB, 2011).

No faltan las voces defensoras de una política más liberal que permita al mercado regularse por sí mismo y aprovechar la libertad como el estudio The Impact of E.U. Internet Privacy Regulations on Early-Stage Investment. A Quantitative Study (Google, 2011) o el trabajo The Impact of Privacy Policy Changes on Venture Capital Investment in Online Advertising Companies (Lerner, 2012).

\section{Metodología}

El objetivo de este estudio se centra en entender cuáles son los ejes del marco jurídico y deontológico de la publicidad comportamental en línea para comprender el debate actual planteado en Europa entre la industria y las instancias políticas. Para ello, desde una perspectiva crítica este estudio expone el contenido y evolución de la normativa, de la interpretación de la misma, y de las iniciativas de autorregulación así como de otros estudios que abordan la regulación y funcionamiento de la publicidad comportamental. 


\section{Regulación jurídica de la publicidad comportamental en línea}

Tanto en Estados Unidos como en la Unión Europa ha habido un interés por regular la publicidad comportamental en línea pero en el intento de conjugar los intereses de la industria y, al mismo tiempo, de salvaguardar los derechos de los usuarios se ha optado por diferentes soluciones. Estados Unidos ha elegido un sistema de autorregulación en el que la empresa informa al usuario de la instalación de cookies y éste puede oponerse a ellas. Es decir, primero hay "un consentimiento tácito informado" (Pérez Bes, 2012: 42) porque las instala y a posteriori si el usuario no las desea puede oponerse. Por el contrario, la Unión Europea se ha decantado por el consentimiento previo del usuario antes de instalar dichas cookies. Es decir, en teoría, el usuario debe ser informado previamente de las cookies y decidir si está de acuerdo o no con su instalación.

La regulación europea es deudora de una especial preocupación por la protección de la privacidad de las comunicaciones electrónicas y de los datos personales de los usuarios de acuerdo con los principios recogidos en la Carta de los Derechos Fundamentales de la Unión Europea en sus artículos 7 (Respeto de la vida privada y familiar) y 8 (Protección de datos de carácter personal). Dicha preocupación queda de manifiesto en la Directiva 1995/46/CE del Parlamento Europeo y del Consejo, de 24 de octubre de 1995, relativa a la protección de las personas físicas en lo que respecta al tratamiento de datos personales y a la libre circulación de esos datos; en la Directiva 2002/58/CE, del Parlamento Europeo y del Consejo, de 12 de julio de 2002, relativa al tratamiento de los datos personales y a la protección de la intimidad en el sector de las comunicaciones electrónicas; y en la reciente Directiva 2009/136/CE, de 25 de noviembre de 2009, por la que se modifican la Directiva 2002/22/CE (sobre el servicio universal), la Directiva 2002/58/CE (sobre la privacidad y comunicaciones electrónicas) y el Reglamento (CE) n..$^{\circ}$ 2006/2004 (sobre la cooperación en materia de protección de los consumidores).

La Directiva 1995/46/CE sobre protección de datos consolida el derecho de los usuarios a acceder, rectificar, cancelar u oponerse al tratamiento de sus datos. Al hilo de estas facultades el artículo 29 promueve la creación de un Grupo de trabajo, el GT $29^{1}$, cuya finalidad es proteger los datos personales y el derecho a la intimidad de los usuarios en el ejercicio de las comunicaciones electrónicas y, en el caso que nos ocupa, en su relación con la publicidad comportamental en línea. Entre sus últimas contribuciones destacan: el Dictamen 2/2010 sobre publicidad comportamental en línea; el Dictamen 16/2011 sobre la recomendación de mejores prácticas de EASA/IAB sobre publicidad comportamental en línea; y el Dictamen 4/2012 sobre la excepción del consentimiento de las cookies.

Por su parte, la Directiva 2002/58/CE, en sus considerandos 24 y 25, alude directamente a las cookies. Se considera que los equipos terminales y la información al-

${ }^{1}$ Las funciones de este Grupo de trabajo (GT29) se describen en el artículo 30 de la Directiva sobre Protección de Datos y en el artículo 14 de la Directiva 97/66/CE del Parlamento Europeo y del Consejo de 15 de diciembre de 1997 relativa al tratamiento de los datos personales y a la protección de la intimidad en el sector de las telecomunicaciones. 
macenadas en ellos son una extensión de la privacidad de los usuarios y, por lo tanto, las cookies o dispositivos similares que se instalan sin consentimiento supone una grave intrusión a la intimidad (considerando 24). De manera que "la información sobre la utilización de distintos dispositivos que se vayan a instalar en el equipo terminal del usuario en la misma conexión y el derecho a impedir la instalación de tales dispositivos se pueden ofrecer en una sola vez durante una misma conexión y abarcar asimismo cualquier posible utilización futura de dichos dispositivos en conexiones posteriores" (considerando 25). Es decir, el usuario tiene el derecho a ser informado y oponerse si así lo considera oportuno, tal y como dispone Directiva 1995/46/CE.

Por último, la reciente Directiva 2009/136/CE en la que se modifica el artículo 5 apartado $3^{2}$ de la Directiva 2002/58/CE dispone que el usuario debe tener información previa de las cookies $\mathrm{y}$, sólo después de haber consentido, deberán proceder a su instalación (opt-in) y solamente con ciertas salvedades: cuando la cookie sea utilizada con el único propósito de efectuar la transmisión de una comunicación por una red de comunicaciones electrónicas; cuando la cookie resulte estrictamente necesaria para la prestación de un servicio de la sociedad de la información expresamente solicitado por el destinatario.

Esta Directiva tenía como plazo para su transposición en los países miembros hasta el 25 de mayo de 2011. España aprobó el Real Decreto-ley 13/2012 que modificaba el artículo 22.2 LSSI (Ley 34/2002, de 11 de julio, de servicios de la sociedad de la información) en relación a las cookies y a los aspectos de la esfera privada de los usuarios, como transposición a esta Directiva. El artículo 22.2 de la LSSI modificado dispone que: "los prestadores de servicios podrán utilizar dispositivos de almacenamiento y recuperación de datos en equipos terminales de los destinatarios, a condición de que los mismos hayan dado su consentimiento después de que se les haya facilitado información clara y completa sobre su utilización." Es decir, el usuario deberá consentir la instalación de las cookies siempre que se les haya dado una información clara y completa sobre su uso. Sin embargo, no queda claro quién tiene la responsabilidad de avisar sobre las cookies a los usuarios, si los editores, los prestadores del servicio o qué otro sujeto, así como tampoco se especifica quién es el responsable del tratamiento los datos personales obtenidos y de la gestión de los mismos o qué sucede si se incumple este artículo. Parece una rápida traducción del artículo sin aclarar ni las responsabilidades ni las consecuencias de su vulneración.

\footnotetext{
"Los Estados miembros velarán por que únicamente se permita el almacenamiento de información, o la obtención de acceso a la información ya almacenada, en el equipo terminal de un abonado o usuario, a condición de que dicho abonado o usuario haya dado su consentimiento después de que se le haya facilitado información clara y completa, en particular sobre los fines del tratamiento de los datos, con arreglo a lo dispuesto en la Directiva 95/46/CE. Lo anterior no impedirá el posible almacenamiento o acceso de índole técnica al solo fin de efectuar la transmisión de una comunicación a través de una red de comunicaciones electrónicas, o en la medida de lo estrictamente necesario a fin de que el proveedor de un servicio de la sociedad de la información preste un servicio expresamente solicitado por el abonado o el usuario."
} 
Las últimas aportaciones del GT 29 han sido decisivas para desarrollar las cuestiones expuestas en la normativa. Sin la intención de analizar exhaustivamente estos documentos, conviene destacar su relevancia de cara a la interpretación y aplicación de las obligaciones legales para la publicidad comportamental en línea. El GT 29 define cookie como todas las tecnologías basadas en el principio de almacenamiento y recuperación de la información en el terminal del usuario. Explica cómo éstas pueden crear dos tipos de perfiles de usuarios: uno, los perfiles predictivos por la navegación de la web y por los anuncios clicados; y, otro, los perfiles explícitos obtenidos a través de los datos proporcionados por los usuarios al registrarse. En lo referente al derecho de los usuarios a decidir sobre la instalación de cookies en sus dispositivos, el GT 29 insta a los proveedores de redes de publicidad a crear mecanismos de autorización previos a dicha instalación. De acuerdo con lo dispuesto en la Directiva 2009/136/CE debe haber un consentimiento jurídicamente efectivo por parte del usuario sobre la instalación de las cookies a priori y no, como se está haciendo, a posteriori.

Asimismo distingue tipologías de cookies y explica cómo aplicar el consentimiento previo de las mismas (opt-out) con algunas excepciones. Resulta indicativo que hace ya más de una década la Recomendación 1/99 sobre el tratamiento invisible y automático de los datos personales en Internet efectuado por software y hardware dispusiera que "en el caso de cookies, debería informarse al usuario cuándo está previsto que el software de Internet reciba, almacene o envíe un cookie. El mensaje debería especificar, en un lenguaje normalmente comprensible, qué información se pretende almacenar en el cookie y con qué objetivo así como el periodo de validez del cookie".

Para conseguir este propósito en la actualidad, el GT 29 entiende que la Administración debe trabajar conjuntamente con la industria dado que actualmente sólo uno de los cuatro principales buscadores bloquea por defecto las cookies de terceros desde el momento en que se instala el buscador, en el resto se aceptan por defecto. Esto supone que se recoge información vulnerando el principio del consentimiento previo. Además, otra premisa imprescindible es que se informe al usuario de qué es una cookie con un lenguaje comprensible, se le advierta de qué información se pretende almacenar en la cookie y se le indique con qué objeto y qué período de validez tiene dicha cookie. Por ello, once años más tarde, el Dictamen 16/2011 anima a la industria a que preste su colaboración en esta materia y cuestiona algunas de las iniciativas de autorregulación en marcha.

Por último, cabe destacar las aportaciones sobre excepción en la prestación del consentimiento para las cookies dispuesto por la Directiva 2009/136/CE y desarrollada por el Dictamen 4/2012. Sólo se permite estas técnicas sin previo aviso cuando haya un fin legítimo como puede ser "el análisis de la efectividad del diseño y de la publicidad insertada en un sitio web o para verificar la identidad de usuarios que intervienen en una transacción en línea" (Pérez Bes, 2012: 44).

El Dictamen 4/2012 desarrolla la interpretación y aplicación de estos requisitos con el objetivo de aclarar si una cookie está exenta o no del consentimiento informado, especificando que el análisis se lleva a cabo sin perjuicio del derecho a ser informado y el derecho a oponerse establecido por la Directiva 95/46/CE. Así se 
permiten ciertas cookies para realizar el seguimiento de entrada de los usuarios al rellenar formularios en línea o para seleccionar la preferencia del idioma.

\section{Autorregulación de la publicidad comportamental en línea}

En la autorregulación de la publicidad comportamental en línea hay que tener presente el carácter pionero de la industria estadounidense en este campo y su influencia en Europa. Por este motivo, al contrario de lo que ocurre en la perspectiva jurídica, la autorregulación de la publicidad comportamental en línea ha sido afrontada con visiones similares tanto en Estados Unidos como en Europa. La autorregulación apuesta por un enfoque más abierto en el que se permite a la industria un amplio margen de maniobra en la elección de la manera de satisfacer los derechos del usuario, punto neurálgico de la legislación europea. Esta perspectiva es la que queda de manifiesto en las iniciativas de autorregulación de la industria europea y en los recientes estudios promovidos por empresas multinacionales.

Desde 1995, en Estados Unidos la Administración y la Comisión Federal del Comercio (FTC) han mostrado una especial preocupación por los derechos de los usuarios en el ejercicio de prácticas empresariales en las que se recolectan datos para ofrecer publicidad personalizada. Con este objetivo la FTC ha desarrollado: informes; mesas de trabajo con los diferentes sectores implicados (industria, consumidores y Administración Pública); y documentos de buenas prácticas, como the Network Advertising Initiative's Self-regulatory Code of Conduct. Estas iniciativas han culminado con la publicación del Informe Staff Report: Self Regulalatory Principles For Online Behavioral Advertising en 2009. Este documento pone de manifiesto los beneficios de la publicidad comportamental en línea y la preocupación del FTC por su problemática relación con la protección de la privacidad de los usuarios.

En el caso de las iniciativas privadas europeas destacan: la Online Behavioural Advertising de EASA/IAB (2011); el estudio elaborado por la multinacional Google The Impact of E.U. Internet Privacy Regulations on Early-Stage Investment A Quantitative Study y el trabajo de Lernet titulado The Impact of Privacy Policy Changes on Venture Capital Investment in Online Advertising Companies.

El Código de Autorregulación Sobre las Buenas Prácticas de la Online Behavioural Advertising de EASA/IAB (2011) fue promovido por la industria e incitado por el Dictamen 16/2011 del GT29 sobre la recomendación de mejores prácticas de EASA/IAB sobre publicidad comportamental en línea. Este Código tiene como objetivo ofrecer a los usuarios la posibilidad de elegir si quieren recibir publicidad comportamental. Para ello proponen que cada vez que el usuario se encuentre en una web con esta publicidad aparezca un icono en el que haciendo clik se redireccione a una web en la que se explica al usario qué es este tipo de publicidad y cuáles son sus implicaciones (www.youronlinechoices.eu). Ese enfoque está muy en la línea de la propuesta de Estados Unidos y ha sido durante criticado por la Unión Europea, específicamente por el GT29 en su Dictamen 4/2012, por no ofrecer al usuario la posibilidad previa de consentir. La cuestión es que "primero se le sigue y después se le informa, contrariamente a lo que disponen la normativa europea" (Martínez Pastor E. y Muñoz Saldaña M., 2012). Además, la signalética del incono no es clara del rastreo 
en la navegación ni del almacenamiento de datos con la finalidad de compartirlos con otras empresas y, posteriormente, ofrecer anuncios personalizados.

Por último, tanto el estudio de Google The Impact of E.U. Internet Privacy Regulations on Early-Stage Investment A Quantitative Study como el estudio de Lerner The Impact of Privacy Policy Changes on Venture Capital Investment in Online Advertising Companies ponen de manifiesto que la reticencia de la industria a la prohibición de investigar los perfiles de los potenciales usuarios en la red. Exponen que ello implicará un descenso significativo de la inversión por parte de los angel bussines en nuevas iniciativas en la red e insisten en que la aplicación estricta de las políticas de privacidad europeas provocará una menor inversión en la industria digital. El resultado de estos estudios manifiesta que la salvaguarda de los derechos de los usuarios por la que apuestan las políticas europeas incidirá directamente en la economía de la red y provocará un retroceso en el desarrollo de la misma.

\section{Conclusiones. Un apunte final}

El mapa que dibuja el panorama jurídico y deontológico evidencia que aún hay mucho camino por recorrer en la concreción y el acuerdo entre las instituciones públicas y la industria sobre de las cuestiones en juego en el ejercicio de la publicidad comportamental en línea. Por un lado, sobre, cuál es la mejor manera de proteger al usuario de la publicidad comportamental ¿políticas opt-out u opt-in? O de dirimir si realmente las cookies vulneran nuestra intimidad dado que no identifican a la persona física, aunque por extensión se entienda que un dispositivo es parte de la privacidad del usuario. La necesidad de encontrar un acuerdo equilibrado entre los intereses públicos y privados es cada vez más urgente. El carácter global de la industria en Internet precisa de una interpretación común, más allá de las fronteras europeas, y de una normativa clara y flexible capaz de aportar algo de luz al farragoso panorama actual. Por otro lado, los legisladores, la industria y las Administraciones Públicas deberían tener presente que en la discusión y soluciones que se adopten sobre las cookies y la publicidad comportamental en línea la gran tarea pendiente es la formación o alfabetización del usuario. Para un alto porcentaje de ciudadanos, la discusión sobre las cookies será probablemente asociada a una "galleta" que nada tiene que ver con la red y, para otros, sencillamente, tendrá que ver con algo que desconocen que les afecta directamente.

El debate jurídico, político y empresarial no puede ser miope al gran problema del desconocimiento de los usuarios sobre el ejercicio de sus derechos en la red y, una vez conocidos dichos derechos, a la posibilidad de posicionarse a favor o en contra de las opciones propuestas, aunque no sean expertos.

\section{Referencias bibliográficas}

BOE (BOLETÍN OFICIAL DEL ESTADO, 2012): Real Decreto-ley 13/2012, de 30 de marzo, por el que se transponen directivas en materia de mercados interiores de electricidad y gas y en materia de comunicaciones electrónicas, y por el que se adoptan medidas para la corrección de las desviaciones por desajustes entre los costes e ingresos de los sectores eléctrico y gasista, BOE núm. 78, de 31 de marzo de 2012, Sec. I. Pág. 26876- 26967. 
EASA/IAB (2011): Código de Autorregulación Sobre las Buenas Prácticas de la Online Behavioural Advertising, en http://www.iabeurope.eu [fecha de consulta: 19 de septiembre de 2012].

FTC (2009): Self-Regulatory Principles For Online Behavioral Advertising, en: http://www.ftc.gov/os/2009/02/P085400behavadreport.pdf [fecha de consulta: 28 de octubre de 2012].

GT 29 (1999), Recomendación 1/99 sobre el tratamiento invisible y automático de datos personales en Internet efectuado por software y hardware, en: http://ec.europa.eu/justice/policies/privacy/docs/wpdocs/1999/wp17es.pdf [fecha de consulta: 20 de septiembre de 2012]

GT 29 (2010), Dictamen 2/2010 sobre publicidad comportamental en línea, en: http://ec.europa.eu/justice/policies/privacy/docs/wpdocs/2010/wp171_es.pdf [fecha de consulta: 25 de septiembre de 2012]

GT 29 (2011), Dictamen 16/2011 sobre la recomendación de mejores prácticas de EASA/IAB sobre publicidad comportamental en línea, en: http://ec.europa.eu/just ice/data-protection/article-29/d ocumentation/opinionrecommendation/files/2011/wp188_es.pdf [fecha de consulta: 4 de octubre de 2012]

GT 29 (2012), Dictamen 4/2012 sobre la excepción del consentimiento de las cookies, en: http://ec.europa.eu/justice/data-protection/article-29/documentation/opinionrecommendation/files/2012/wp194_en.pdf [fecha de consulta: 2 de noviembre de 2012].

LERNER, Joshua (2012): "The Impact of Privacy Policy Changes on Venture Capital Investment in Online Advertising Companies", en: http://www.analysisgroup.com/uploadedFiles/Publishing/Articles/Lerner_Fall2011 Copyright Policy _VC_Investments.pdf [fecha de consulta: 30 de octübre de 2012].

MARTÍNEZ PASTOR, Esther y MUÑOZ SALDAÑA, Mercedes (2012): "La regulación de la publicidad en las redes sociales", en NICOLÁS, Miguel Ángel y GRANDÍO, María del Mar: Estrategia de comunicación en redes sociales. Barcelona, Gedisa, pp. 83-100.

PÉREZ BES, Francisco (2012): Publicidad comportamental online. Barcelona. UOC.

UNIÓN EUROPEA (1995): Directiva 95/46/CE del Parlamento Europeo y del Consejo, de 24 de octubre de 1995, relativa a la protección de las personas físicas en lo que respecta al tratamiento de datos personales y a la libre circulación de estos datos. Diario Oficial $n^{\circ}$ L 281 de 23/11/1995 p. 0031 - 0050- DO L 281 de 23/11/1995 pp. $0031-0050$.

UNIÓN EUROPEA (2002): Directiva 2002/58/CE del Parlamento Europeo y del Consejo, de 12 de julio de 2002, relativa al tratamiento de los datos personales y a la protección de la intimidad en el sector de las comunicaciones electrónicas (Directiva sobre la privacidad y las comunicaciones electrónicas) DO L 201 de 31/07/2002 pp. $0037-0047$. 
UNIÓN EUROPEA (2009): Directiva 2009/136/CE, por la que se modifican la Directiva 2002/22/CE relativa al servicio universal y los derechos de los usuarios en relación con las redes y los servicios de comunicaciones electrónicas, la Directiva 2002/58/CE relativa al tratamiento de los datos personales y a la protección de la intimidad en el sector de las comunicaciones electrónicas, DO L 337 de 18/12/2009, pp. 0011-0036.

UNIÓN EUROPEA (2010): Carta de los Derechos Fundamentales de la Unión Europea, DO C 83, de 30.03.2010, pp. 389-403.

\section{Esther MARTÍNEZ PASTOR}

Universidad Rey Juan Carlos de Madrid

Profesora Contratada Doctora

esther.martinez.pastor@urjc.es

\section{Mercedes MUÑ̃Z SALDÃ̃̃}

Universidad de Navarra

Departamento de Comunicación Pública

Profesora Contratada Doctora

mms@unav.es 